\title{
Dissemination and experience with cognitive processing therapy
}

\author{
Kathleen M. Chard, PhD; ${ }^{1-2 *}$ Elizabeth G. Ricksecker, MA; ${ }^{1}$ Ellen T. Healy, PhD; ${ }^{3}$ Bradley E. Karlin, PhD; ${ }^{4}$ \\ Patricia A. Resick, PhD $^{3,5}$ \\ ${ }^{1}$ Cincinnati Department of Veterans Affairs (VA) Medical Center, Cincinnati, OH; ${ }^{2}$ University of Cincinnati College of \\ Medicine, Cincinnati, OH; ${ }^{3}$ National Center for Posttraumatic Stress Disorder, VA Boston Healthcare System, Boston, \\ MA; ${ }^{4}$ Office of Mental Health Services, VA Central Office, Washington, DC; ${ }^{5}$ Boston University School of Medicine, \\ Boston, $M A$
}

\begin{abstract}
Clinical practice guidelines suggest that cognitive behavioral therapies are recommended for the treatment of posttraumatic stress disorder (PTSD). One of these treatments, cognitive processing therapy (CPT), is an evidence-based treatment that has been shown to be effective at treating combat, assault, and interpersonal violence trauma in randomized controlled trials. The Department of Veterans Affairs (VA) Office of Mental Health Services has implemented an initiative to disseminate CPT as part of a broad effort to make evidence-based psychotherapies widely available throughout the VA healthcare system. This article provides an overview of CPT and reviews the efficacy and program evaluation data supporting its use in a variety of settings. In addition, we report on survey data from individuals who have participated in the VA initiative and on outcome data from patients treated by rollout-trained therapists. Our data suggest that many clinicians trained in the rollout show good adoption of the CPT model and demonstrate solid improvements in their patients' PTSD and depressive symptomotology. Finally, we offer recommendations for using CPT in clinical settings.
\end{abstract}

Key words: cognitive processing, cognitive processing therapy, dissemination, evidence-based psychotherapy, military, posttraumatic stress disorder, program evaluation, survey, trauma, Veterans.

\section{INTRODUCTION}

The Department of Veterans Affairs (VA) and Department of Defense (DOD) VA/DOD Clinical Practice
Guideline for Management of Post-Traumatic Stress identifies treatment recommendations for clinicians in VA and DOD treatment settings based on an extensive literature review of the current evidence supporting the treatments for posttraumatic stress disorder (PTSD). The guideline strongly recommends evidence-based, traumafocused psychotherapies (e.g., cognitive processing therapy [CPT] and prolonged exposure [PE]) as the first-line treatment options for patients with PTSD.

Abbreviations: BDI-II = Beck Depression Inventory-II, CBW = Challenging Beliefs Worksheet, $\mathrm{CPT}=$ cognitive processing therapy, CPT-C = CPT-Cognitive Only, CPT-SA = CPT-Sexual Assault, CQW = Challenging Questions Worksheet, DOD = Department of Defense, DSM-IV = Diagnostic and Statistical Manual of Mental Disorders-Fourth Edition, EBP = evidencebased psychotherapy, ITT $=$ intent-to-treat, $\mathrm{MA}=$ minimal attention, $\mathrm{OEF}=$ Operation Enduring Freedom, OIF = Operation Iraqi Freedom, PCL $=$ Posttraumatic Stress Disorder Checklist, $\mathrm{PE}=$ prolonged exposure, $\mathrm{PTSD}=$ posttraumatic stress disorder, $\mathrm{RCT}=$ randomized controlled trial, $\mathrm{T} 1=$ initial survey, $\mathrm{T} 2$ = follow-up survey, TBI = traumatic brain injury, VA = Department of Veterans Affairs, WA = written account.

*Address all correspondence to Kathleen M. Chard, PhD; PTSD \& Anxiety Disorders Clinic, Cincinnati VA Medical Center, 1000 S Ft. Thomas Ave, Ft. Thomas, KY 41075; 859-572-6208; fax: 859-572-6748.

Email: Kathleen.Chard@va.gov

http://dx.doi.org/10.1682/JRRD.2011.10.0198 
On the national VA level, there have been programmatic initiatives to help promote the implementation of evidence-based psychotherapies (EBPs) that have had a positive effect on the CPT dissemination initiative and implementation of CPT within VA. For example, local EBP coordinators have been placed at each VA medical center to serve as an advocate and clinical champion for EBPs at the local level and to promote clinical infrastructures (e.g., 60-90 min weekly clinics) that support the delivery of EBPs. Local EBP coordinators disseminate information about empirically supported treatments, help facilitate the local recruitment of clinicians to be trained in CPT and other EBPs, and serve as a local advocate to troubleshoot barriers to implementation and promote sustainability. Throughout VA, emphasis on EBP implementation has significantly increased, with facility-specific performance measures and metrics to track implementation. These efforts reinforce the goals of the CPT initiative and help promote a consistent message as to the importance of including EBP in the treatment options for Veterans with PTSD.

\section{TREATMENT OVERVIEW}

CPT is a predominantly cognitive treatment for individuals with PTSD and related symptomatology, such as depression, anxiety, and guilt [1]. CPT consists of 12 weekly sessions delivered in a manualized, serial format. CPT can be delivered in group, individual, or combined group and individual formats. In sessions 1 through 4, patients are educated regarding the theory behind CPT and asked to explore the "meaning" of their traumas by writing an impact statement discussing why they believe the traumatic event occurred and how the event has shaped their beliefs about self, others, and the world, particularly in the areas related to safety, trust, power/control, esteem, and intimacy. Next, patients are taught the connection between events, thoughts, and feelings through the use of the A-B-C Sheet, and together with the therapist, they begin to identify places where the patients have become "stuck" in their thinking. Specifically, they learn to identify "stuck points," which are thoughts related to interpretations of their traumatic event, such as "It was my fault the trauma happened," or thoughts of how they view themselves and the world now based on their traumatic experiences, such as "I can't trust anyone" and "I am worthless.” Finally, the patients write detailed accounts of the most traumatic incident, including sensory details, thoughts, and feelings. (Note: in the CPT-Cognitive Only [CPT-C] version, no traumatic accounts are written.) At the same time, the therapist uses Socratic dialogue to help the patients begin to analyze their stuck points and to view past, present, and future events with a more balanced interpretation.

In sessions 5 through 7 , the core cognitive therapy skills are taught, including use of the Challenging Questions Worksheet (CQW) to examine a single belief. The CQW consists of ten questions that help the Veterans evaluate their stuck points from a number of angles, including looking at the evidence for and against the belief, examining the context from which the belief was formed, and identifying how much the belief is based on feelings rather than thoughts, for example. In session 6 , the Patterns of Problematic Thinking worksheet is introduced to allow patients to become familiar with common faulty thinking patterns that can interfere with recovery from PTSD. The Veteran examines each stuck point to see which of seven patterns are being activated, such as overgeneralizing from a single incident, thinking in terms of black/white or right or wrong, or emotional reasoning. Finally, the Challenging Beliefs Worksheet (CBW) is introduced, which incorporates all of the prior worksheets and allows patients to not only challenge their beliefs but also to identify more realistic and balanced beliefs and their related emotions.

In sessions 8 through 12, using the CBW, patients focus their thought examination in each of five key areas, including safety, trust, power/control, esteem, and intimacy. In session 12, the patients rewrite their impact statement and compare it with the version written at the beginning of the therapy. This allows the patients to clearly recognize the changes in their thoughts, feelings, and behaviors. Finally, the therapist and patients look to the future and identify any areas that may continue to be problematic for the patients and discuss ways that they can manage these issues using the CPT principles.

When CPT or CPT-C are administered in group, the same session structure is used either in the group only or group and individual combined formats. In neither case are the trauma accounts discussed in group, thus reducing the likelihood of secondary traumatization occurring, and allowing the group focus to remain on moving toward healthier thinking patterns. If patients write their trauma accounts, the therapist reviews them out of session and provides written feedback to the patient with support, 
questions, and suggestions for areas that might contain stuck points. When group and individual therapies are combined, the patients can read their trauma accounts in the individual session as outlined in the clinical practice guidelines.

\section{EVIDENCE BASE FOR COGNITIVE PROCESS- ING THERAPY}

The efficacy and effectiveness of CPT has been demonstrated in a wide range of traumatized populations, including female rape victims, childhood sexual abuse survivors, and combat Veterans. Using a sample of female rape victims, Resick et al. compared CPT with PE and a minimal attention (MA) control group in a randomized controlled trial (RCT) [2]. Analysis of the intent-totreat (ITT) sample, which included those who never started the treatment and those who dropped out, demonstrated that both CPT and PE led to significant reductions in PTSD and depressive symptoms posttreatment (CPT vs MA: $g=0.84-1.22$; PE vs MA: $g=0.57-0.74$ ) and at 3- and 9-month follow-up. Additional analyses indicated that CPT resulted in small but positive effect-size differences for PTSD, depression, and guilt measures at posttreatment, 3 months, and 9 months, indicating modestly greater symptomatic improvement relative to the participants in the PE condition. Monson et al. conducted an RCT comparing CPT with a wait-list control in Veterans, primarily Vietnam war Veterans who had chronic military-related PTSD [3]. The Veterans who received CPT had a significant reduction in severity of PTSD symptoms over time as compared with the wait-list control group $(g=1.01-1.12)$. Of the ITT sample, 40 percent did not mot meet criteria for PTSD after engaging in CPT treatment.

CPT has also been shown to be efficacious with samples of childhood sexual abuse survivors [4]. Chard studied an adaptation of CPT (CPT-Sexual Assault [CPT-SA]) for survivors of sexual assault that combined individual and group treatment. In this study, CPT-SA was compared with a wait-list control group in an RCT. For those in the treatment group, symptoms of PTSD $(d=1.52$ $1.55)$, depression $(d=1.42)$, and dissociation $(d=0.91)$ significantly declined at posttreatment and continued to decline at 3-month follow-up. Treatment gains were maintained when assessed at 1-year follow-up.
A dismantling study was conducted to better understand how the components of CPT contributed to treatment outcome in a sample of adult women with a history of sexual or physical assault [5]. In an RCT, the full protocol of CPT was compared with a CPT-C condition that excluded the trauma written account (WA) and a trauma WA-only condition. For CPT and CPT-C groups, treatment sessions were held twice weekly for $50 \mathrm{~min}$; for the WA group, after the first week sessions were held once per week for 2 hours over the 6-week treatment. Contrary to the hypothesis that the full CPT protocol would lead to superior treatment outcomes over the component conditions, participants in all three treatment groups demonstrated significant decline in PTSD symptoms posttreatment (CPT: $g=1.1$; CPT-C: $g=1.1$; WA: $g=$ 0.7 ) and at follow-up (CPT: $g=1.2$; CPT-C: $g=1.1$; WA: $g=1.0$ ). Furthermore, over the course of treatment, the CPT-C group had significantly larger reductions in PTSD than the WA condition and achieved clinically significant reductions in PTSD symptoms faster than either the CPT or WA groups. Overall, the outcomes for the CPT and CPT-C conditions did not differ significantly. Resick et al. concluded that while the WA condition had good outcomes, the CPT-C condition did even better, with both $\mathrm{CPT}$ and CPT-C appearing to be comparably effective in treating PTSD [5]. Thus, it was suggested that the trauma WA may not be a necessary component for all patients, though some may benefit by writing their trauma account if they need to reconstruct the event and/or access emotions that have been avoided.

In addition to RCTs, CPT has also been examined extensively in clinical settings with Veterans. In an outpatient setting, both Vietnam war and Operation Iraqi Freedom (OIF) and Operation Enduring Freedom (OEF) Veterans receiving CPT demonstrated significant drops in PTSD and depressive symptoms from pre- to posttreatment [6]. In another study, male patients with comorbid PTSD and traumatic brain injury (TBI) who received CPT-C, augmented by psychoeducation groups, speech therapy, and cognitive retraining, in a PTSD and TBI residential setting showed significant improvements in PTSD symptoms [7]. In this study, 28 male patients with a history of mild TBI were compared with 14 male patients with a history of moderate or severe TBI, and results showed that although both groups improved from pre- to posttreatment, patients with a moderate or severe TBI showed significantly more improvement in PTSD and depressive symptoms than patients with mild TBI. 
Finally, a recent study by Alvarez et al. demonstrated that group CPT in a male residential program was superior to trauma-focused group treatment as usual with respect to PTSD and depressive symptoms, coping, and psychological distress [8]. Given the evidence that CPT is effective, it is important to train clinicians so this treatment is available to patients with PTSD.

\section{METHODS}

\section{VA Cognitive Processing Therapy Training Initiative}

As part of its efforts to make EBPs widely available to Veterans, VA has established a national policy that requires that all Veterans with PTSD have access to CPT or PE. To that end, in 2006, VA established a national CPT training initiative with the goal of disseminating and implementing CPT throughout the VA healthcare system (see Karlin et al. [9]). As of August 31, 2011, the CPT rollout trainers have provided training to over 3,000 mental health staff through 61 rollout and 59 nonrollout workshops. In addition, the trainers have provided CPT training to over 2,400 mental health clinicians in DOD and over 400 mental health clinicians in the community. In the VA rollout, the training process for clinicians includes attending a 3-day workshop and then participating in 6 months of weekly telephone consultation with one of the expert CPT trainers or consultants. Clinicians must complete at least two CPT individual cases or one CPT group while participating in consultation. After completing the training requirements, they can formally apply to be designated as a "CPT provider." Initial program evaluation data described elsewhere [9] have indicated that the training and implementation of CPT has yielded significant and positive therapist, patient, and system outcomes. Next, we report on expanded program evaluation data.

\section{Workshop Training Evaluation Surveys}

As part of our efforts to monitor implementation, we collected two sets of surveys, the posttraining evaluation survey and the pre- and postworkshop clinical self-efficacy therapist survey, from VA mental health clinicians who attended the CPT training workshops.

\section{Posttraining Evaluation Survey}

For continuing education unit accreditation purposes, the posttraining evaluation survey is administered at the conclusion of all formal rollout training program workshops. This survey includes a self-report assessment of the faculty members conducting the workshop, achievement of program objectives, and participant satisfaction with the CPT training. The items designed to assess the achievement of program objectives are rated on a 5-point scale ranging from "completely" to "not at all." The items designed to assess the faculty and participant satisfaction are rated on a 4-point scale ranging from "strongly agree" to "strongly disagree."

\section{Pre- and Postworkshop Clinical Self-Efficacy Therapist} Survey

Since April 2010, the VA CPT training program has also administered pre- and postworkshop therapist surveys designed to evaluate clinician self-efficacy in delivering CPT-specific skills and attitudes toward the CPT therapy. Clinician self-efficacy items are rated on a 6-point scale ranging from "not confident at all" to "extremely confident," and the items measuring a clinician's attitudes toward the CPT therapy are rated on a 5-point scale ranging from "strongly disagree" to "strongly agree."

\section{Cognitive Processing Therapy Practice Survey}

Early in the dissemination, we decided to conduct an informative process evaluation of the national rollout and incorporate salient programmatic changes based on the data collected from participants. The goal was to examine how clinicians are using CPT in their clinical practice, to identify the barriers to CPT implementation, and to assess the effectiveness of program administration refinement methods employed to address those barriers and for improving our ongoing dissemination efforts.

Thus, in March 2008, an initial CPT Practice Survey was sent to all VA mental health clinicians who attended any of the CPT national rollout training workshops held between July 2007 and March 2008. The anonymous online survey was developed and administered through the Inquisite Survey (Allegiance Inc; Austin, Texas) Internet software system, and CPT workshop attendees were invited to participate in the survey through email notifications. We solicited information from clinicians who had begun providing the therapy as well as from those who had not yet adopted CPT in their clinical practice. In addition to demographic and work-setting elements, the survey instrument contained items designed to assess various CPT caseload and service delivery components, aspects that hinder implementation and prevent clinicians from providing CPT within their clinical settings, 
the use of postworkshop consultation calls, and adherence to treatment protocol fidelity.

From an analysis of the initial survey (T1), we identified key implementation issues for program refinement. As will be discussed in detail in the "Refinements to Rollout" section, these included addressing the significant barriers to implementation, such as clinicians not having enough time in their schedule to provide CPT to more patients, as well as their workload being too heavy to accommodate attendance on consultation calls and providing CPT to more patients. In addition, program enhancements were also made to address the consultation call scheduling issues identified, such as expanding the number of calls available and modifying the scheduling procedures to facilitate attendance.

In February 2011, we sent a follow-up survey (T2) to the same cohort of VA mental health clinicians trained between July 2007 and March 2008 for comparison purposes in an effort to determine the effect of the programmatic changes instituted. We developed and administered the anonymous online follow-up survey through the SurveyMonkey (Portland, Oregon) Internet software system, and CPT workshop attendees were once again invited to participate through email notifications.

Also during February 2011, in addition to collecting follow-up data from those previously surveyed, we sent an initial CPT Practice Survey to all VA mental health clinicians who attended any of the CPT national rollout training workshops held between January 2009 and December 2010. This survey was administered as the baseline data for this cohort of clinicians, as well as for comparison purposes to the $2008 \mathrm{~T} 1$ cohort, to further determine the effect of the programmatic changes that were instituted. The anonymous online survey was administered through SurveyMonkey, and CPT workshop attendees were once again invited to participate through email notifications. As with the $2008 \mathrm{~T} 1$ and T2, information was again solicited from clinicians who had begun providing the therapy as well as from those who had not yet adopted CPT in their clinical practice. In addition to the same demographic and work-setting elements, the $2011 \mathrm{~T} 1$ also contained items designed to assess various CPT caseload and service delivery components, aspects that hinder implementation and prevent clinicians from providing CPT within their clinical settings, the use of postworkshop consultation, and adherence to treatment protocol fidelity.

\section{Measuring Patient Outcomes}

The main goal of CPT is to reduce the patient's psychological distress caused by the symptoms of PTSD and related comorbid disorders. As a core component of the CPT dissemination initiative, evaluation efforts to assess patient clinical outcomes have also been implemented to determine the effect and demonstrate the effectiveness of the program in routine care conditions and practice settings. These patient outcome data are collected during the consultation phase of the training program and include the total score for the Posttraumatic Stress Disorder Checklist (PCL) [10] and, when possible, the Beck Depression Inventory-II (BDI-II) [11].

The weekly version of the PCL is a standard outcome measure in the CPT protocol for assessing patient selfreported PTSD symptoms. The PCL consists of 17 items corresponding to the PTSD symptom criteria specified in the Diagnostic and Statistical Manual of Mental Disorders-Fourth Edition (DSM-IV) [12]. Items on the PCL are rated based on how much they bothered the patient on a 5-point scale, where $1=$ not at all and 5 = extremely, yielding a total score ranging from 17 to 85 . Among samples of Veterans, the PCL has demonstrated excellent internal consistency and test-retest reliability, as well as strong convergent and discriminant validity [10].

The BDI-II is an optional outcome measure in CPT, used when relevant to assess patient self-reported depression symptoms. The BDI-II is a 21-item self-report instrument that measures the severity of depression over the preceding 2 weeks. Items on the BDI-II are scored on a 4-point scale from 0 to 3 , yielding a total score ranging from 0 to 63 . The BDI-II, which is in line with the depression criteria of the DSM-IV [12], has demonstrated excellent internal consistency and test-retest reliability along with strong convergent validity [13].

\section{RESULTS}

\section{Workshop Training Evaluation Surveys}

\section{Posttraining Evaluation Survey}

Since January 2010, posttraining evaluation data have been collected from 448 participants at 14 CPT rollout training program initiative workshops. Of those who responded, more than 90 percent of workshop participants reported either "complete" or "mostly complete" achievement of all CPT training program objectives. In 
addition, 96.2 percent of clinicians indicated "completely" or "mostly complete" with respect to their ability to describe PTSD symptoms and the theory underlying CPT following the training, and 91.2 percent reported that, following the training, they have acquired "complete" or "mostly complete" knowledge and skills required to implement CPT with Veterans diagnosed with PTSD. Further, the overwhelming majority of clinicians surveyed indicated that the teaching strategies used in the CPT training program were appropriate (mean \pm standard deviation: $3.67 \pm 0.09$ ), the faculty effectively communicated information (3.77 \pm 0.12 ), they developed new skills and knowledge as a result of their participation in the workshop (3.67 \pm 0.12 ), and the program was worthwhile (3.75 \pm 0.09$)$.

\section{Pre- and Postworkshop Clinical Self-Efficacy Therapist Survey}

The pre- and postworkshop therapist survey evaluation data have been collected from 320 participants at 11 CPT rollout training program initiative workshops since April 2010. To investigate change over time, we used repeated-measure $t$-tests to compare pre- and postworkshop data. Following the training, clinicians indicated a significantly higher level of confidence and greater degree of self-efficacy on all aspects of CPT-specific skills and the various structured assignment components of therapy implementation. Among these items were "use Socratic dialogue to help patients expand their perspective" (paired $t(279)=13.67, p<0.001$ ), "elicit and focus on patients' specific cognitions or stuck points relevant to their trauma account" (paired $t(279)=17.62, p<0.001$ ), "assign and review an Impact Statement to explore the meaning of the trauma and identify stuck points" (paired $t(279)=18.42, p<0.001)$, and "assign the [CBW] to assist the patient in examining stuck points and identifying more balanced ways of interpreting situations" (paired $t(273)=20.78, p<0.001)$. Clinicians also reported a significant increase in their ability to both explain CPT to patients (paired $t(275)=19.57, p<0.001$ ) and reduce symptoms of PTSD by using CPT (paired $t(274)=19.94, p<0.001)$.

Clinician attitudes toward the utility and effectiveness of CPT were also significantly higher following the workshop training. Included among these items were "adherence to the CPT protocol increases patient satisfaction with therapy" (paired $t(273)=11.98, p<0.001$ ), "close adherence to the CPT protocol improves patient outcomes" (paired $t(273)=9.78, p<0.001$ ), and "CPT is an effective treatment for most patients visiting outpatient PTSD clinics" (paired $t(273)=8.52, p<0.001$ ). Furthermore, following the workshop, clinicians also indicated a significant decrease in their responses to the item "close adherence to 'evidence-based therapy' modalities increases therapist burnout" (paired $t(273)=$ 2.08, $p<0.001$ ).

The workshop survey results suggest that attendees are highly satisfied with the quality of the workshop training and they report that they have developed new clinical skills as a result of their participation. Finally, attendees endorse a greater understanding of CPT and the effectiveness of the treatment protocol and report that they have acquired increased confidence regarding their therapeutic skill level and ability to provide CPT to their patients.

\section{Cognitive Processing Therapy Practice Survey}

In March 2008, an initial CPT Practice Survey was sent to all VA mental health clinicians who attended any of the CPT national rollout training workshops held between July 2007 and March 2008. Of the 753 clinicians we contacted, 325 completed $\mathrm{T} 1$, providing a response rate of 43.2 percent. The majority of clinicians who completed T1 were female (67.1\%), and most respondents indicated that they were either a psychologist $(44.0 \%)$ or a social worker (40.6\%). In addition, their primary VA work setting was either a mental health clinic (51.5\%) or a PTSD clinic (39.2\%). With regard to implementation, among those survey respondents who have started using CPT, the most common format used with patients was individual CPT (81.7\%), and the majority of clinicians reported that they would like to be using CPT with more patients (88.8\%).

During February 2011, T2 was sent to the same cohort of VA mental health clinicians trained between July 2007 and March 2008 for comparison purposes to assess the effect of the programmatic changes instituted. Out of these 753 clinicians, 111 no longer had working VA email addresses and were lost to follow-up. Of the remaining 642 clinicians contacted, 237 completed T2, providing a response rate of 34.1 percent.

As was the case with $\mathrm{T} 1$, the majority of clinicians who completed T2 were female (68.9\%), and most respondents indicated that they were either a psychologist (52.2\%) or a social worker (39.0\%). In addition, their primary VA work setting was either a mental health clinic 
(23.2\%) or a PTSD clinic (41.1\%). With regard to implementation, among those survey respondents who have started using CPT, the most common format of CPT used with patients was individual CPT (72.6\%), and the majority of clinicians reported that they would like to be using CPT with more patients (70.8\%). There were no significant differences between the group of clinicians who completed T1 and those who completed T2.

When examining the change in data from $\mathrm{T} 1$ to $\mathrm{T} 2$, we found several significant results. We observed substantial reductions in all of the most frequently reported reasons for not starting CPT with more patients, barriers to using CPT with more patients, and not attending CPT consultation calls. With respect to the reasons for not starting CPT with more patients, the two most frequently reported items were "having no or little room in their schedule" (T1 $=52.0 \%, \mathrm{~T} 2=8.2 \%)$ and "workload is too heavy" (T1 $=44.0 \%, \mathrm{~T} 2=8.7 \%)$. The endorsement of both items was significantly reduced at follow-up $\left(\chi^{2}=\right.$ 38.78, $p<0.001$, and $\chi^{2}=25.97, p<0.001$, respectively). When examining the data regarding the barriers for not using CPT with more patients, again the two most frequently reported items at T1 were "having no or little room in their schedule" (T1 $=55.4 \%, \mathrm{~T} 2=38.4 \%)$ and "workload is too heavy" ( $11=54.9 \%, \mathrm{~T} 2=36.5 \%)$. Likewise, the frequency of clinician endorsement on both was significantly reduced at follow-up $\left(\chi^{2}=12.04, p<\right.$ 0.001 , and $\chi^{2}=14.01, p<0.001$, respectively). Finally, the two most frequently reported reasons for not attending consultation calls were "workload is too heavy" (T1 = $66.9 \%, \mathrm{~T} 2=20.5 \%$ ) and "not available during call times provided" ( $\mathrm{T} 1=18.5 \%$, $\mathrm{T} 2=7.8 \%)$. Once again, the endorsement of both of these items by clinicians were also significantly reduced at follow-up $\left(\chi^{2}=72.83, p<\right.$ 0.001 , and $\chi^{2}=8.94, p<0.01$, respectively). Because both the initial and follow-up CPT Practice Survey administrations were anonymous, conducting a repeatedmeasures analysis of the data collected from the subset of therapists who completed both T1 and T2 was not possible.

Also during February 2011, in addition to collecting follow-up data from those previously surveyed, we sent an initial CPT Practice Survey to all VA mental health clinicians who attended any of the CPT national rollout training workshops held between January 2009 and December 2010. This survey was administered as the baseline data for this cohort of clinicians, as well as for comparison purposes to the $2008 \mathrm{~T} 1$ cohort, to further determine the effect of the programmatic changes that were instituted. Of these 1,153 clinicians, 46 were lost to follow-up and 541 of the remaining 1,107 clinicians completed the survey, providing for a response rate of 48.9 percent.

As was the case with both the $2008 \mathrm{~T} 1$ and T2, the majority of clinicians who completed the $2011 \mathrm{~T} 1$ were female $(68.2 \%)$, were either a psychologist $(42.1 \%)$ or social worker $(50.4 \%)$, and reported that their primary VA work setting was a community-based outpatient clinic (29.2\%) or PTSD clinic (24.6\%). In addition, among those survey respondents who have started using CPT, the most common format of CPT used with patients was individual CPT (73.9\%), and the majority of clinicians would like to be using CPT with more patients (70.8\%). There were no significant differences between the group of clinicians who completed the $2008 \mathrm{~T} 1$ and T2 and those who participated in the $2011 \mathrm{~T} 1$.

In a similar fashion to the $2008 \mathrm{~T} 1$ versus T2 analyses reported earlier, the $2011 \mathrm{~T} 1$ cohort data were also compared with the $2008 \mathrm{~T} 1$ cohort data to assess the effect that the dissemination program improvements have made over time regarding the previously identified barriers to implementation. When comparing the responses to the $2008 \mathrm{~T} 1$ and the $2011 \mathrm{~T} 1$, we again observed positive changes in the perceived barriers to using CPT. For example, clinicians responding to the $2011 \mathrm{~T} 1$ were significantly less likely to endorse barriers to starting CPT with more patients, barriers to using CPT with more patients, and reasons for not attending CPT consultation calls, as had been reported at the $2008 \mathrm{~T} 1$. With regard to the reasons for not starting CPT with more patients, the two most frequently reported items, "having no or little room in their schedule" $(2008 \mathrm{~T} 1=52.0 \%, 2011 \mathrm{~T} 1=$ $6.5 \%)$ and "workload is too heavy" (2008 T1 $=44.0 \%$, $2011 \mathrm{~T} 1=6.7 \%$ ), were both significantly reduced at 2011 $\mathrm{T} 1\left(\chi^{2}=63.83, p<0.001\right.$, and $\chi^{2}=43.77, p<0.001$, respectively). When examining the data regarding the barriers for not using CPT with more patients, the two most frequently reported items by the $2008 \mathrm{~T} 1$ cohort were again "having no or little room in their schedule" (2008 T1 $=55.4 \%, 2011 \mathrm{~T} 1=36.4 \%)$ and "workload is too heavy" (2008 T1 $=54.9 \%, 2011 \mathrm{~T} 1=36.0 \%)$. The endorsement of both items by the $2011 \mathrm{~T} 1$ cohort was also significantly less frequent $\left(\chi^{2}=21.22, p<0.001\right.$, and $\chi^{2}=20.96, p<0.001$, respectively). Finally, with respect to the reasons for not attending consultation calls, the two most frequently reported items were "workload is too heavy" (2008 T1 = 66.9\%, $2011 \mathrm{~T} 1=8.7 \%$ ) and "not 
available during call times provided" $(2008 \mathrm{~T} 1=18.5 \%$, $2011 \mathrm{~T} 1=3.1 \%)$. In addition to these items being among the least reported barriers by clinicians completing the $2011 \mathrm{~T} 1$, they were also endorsed significantly less often than the 2008 T1 cohort $\left(\chi^{2}=217.62, p<0.001\right.$, and $\chi^{2}=$ $42.35, p<0.001$, respectively).

\section{Patient Outcomes}

Patient clinical outcome data are currently available on 374 Veterans treated by therapists participating in the rollout after their first two CPT cases that have completed the full treatment protocol. Of these Veterans, 86 percent were male and 14 percent were female. The majority were Caucasian (73\%), 18 percent were African American, and only 5 percent were of Hispanic origin. More than half were married (60\%), 24 percent were divorced, and 12 percent were single. The majority had either a high school education (44\%) or completed some college (34\%). The mean age of this group was $50 \mathrm{yr}$. In addition, 40 percent of the Veterans served in the Vietnam war, 29 percent served in OIF/OEF, and 12 percent served in the Persian Gulf war. The majority of these Veterans received the CPT treatment protocol (73\%), while 27 percent received the CPT-C protocol. Furthermore, 64 percent received individual therapy sessions, 30 percent participated in group therapy, and only 6 percent received a combination of individual and group sessions during treatment.

Overall, the pretreatment PCL score was $64.1 \pm$ 10.71 and the posttreatment PCL score was $45.2 \pm 14.13$ (Figure 1). This 18.9-point change in PCL score was clinically meaningful and statistically significant (paired $t(373)=28.22, p<0.001)$ and indicated a 29.5 percent reduction in self-reported PTSD symptoms. Furthermore, 9.9 percent of the patients had a PCL score below the standard clinical PTSD cutoff score of 50 at initial session, while 59.4 percent had a PCL score below the clinical PTSD cutoff score of 50 at the final session. When these same data were examined by conflict era, similar patterns of improvement in PTSD symptoms were demonstrated and the reduction in PCL scores were statistically significant for Vietnam war Veterans (paired $t(148)=$ 16.40, $p<0.001$ ), OIF/OEF Veterans (paired $t(106)=$ $16.42, p<0.001$ ), and Persian Gulf war Veterans (paired $t(43)=10.85, p<0.001)$.

Pre- and posttreatment BDI-II scores were available for 53 of the 374 Veterans who completed CPT. For the overall group, the pretreatment BDI-II score was $30.9 \pm$
10.96, which indicates a severe level of depression (standard clinical cutoff score range is 29-63), and the posttreatment BDI-II score was $19.7 \pm 10.13$ (Figure 2), which indicates a mild level of depression (standard clinical cutoff score range is 14-19). This 11.2-point change in BDI-II score was clinically meaningful and statistically significant (paired $t(52)=9.24, p<0.001$ ) and indicated a 36.5 percent reduction in self-reported depression symptom severity. When these same data were examined by conflict era, similar patterns of improvement in depression symptom severity were demonstrated and the reduction in BDI-II scores were statistically significant for Vietnam war Veterans (paired $t(19)=5.35, p<$ 0.001), OIF/OEF Veterans (paired $t(19)=5.66, p<$ 0.001 ), and Persian Gulf war Veterans (paired $t(6)=4.02$, $p<0.01)$.

\section{DISCUSSION}

\section{Refinements to Rollout}

We believe many of the positive changes observed between the two surveys were caused by specific efforts at both program administration and clinical and/or teaching levels. For example, the commitment required by training attendees to participate in dissemination processes was further emphasized and clarified, and clinician workshop applicants and their supervisors were required to sign a training agreement prior to the clinician being accepted into the CPT program. This agreement served to document their commitment to following through with the training requirements after attending the workshop, which include completing CPT cases and attending weekly consultation calls. Additionally, the structure of the consultation calls was modified so that all training participants were assigned to a regular weekly call with the same training consultant each week. This change promoted more consistency in consultation because consultants were able to follow each caller's cases. Because of high demand, the number of consultation call times available was significantly increased; currently, 58 calls are held each week. Finally, decentralized CPT training processes have been implemented to provide training capacity (i.e., regional trainers and consultants) in each Veterans Integrated Service Network and Vet Center region, as well as DOD. The goal is to make CPT training more widely available with more CPT 


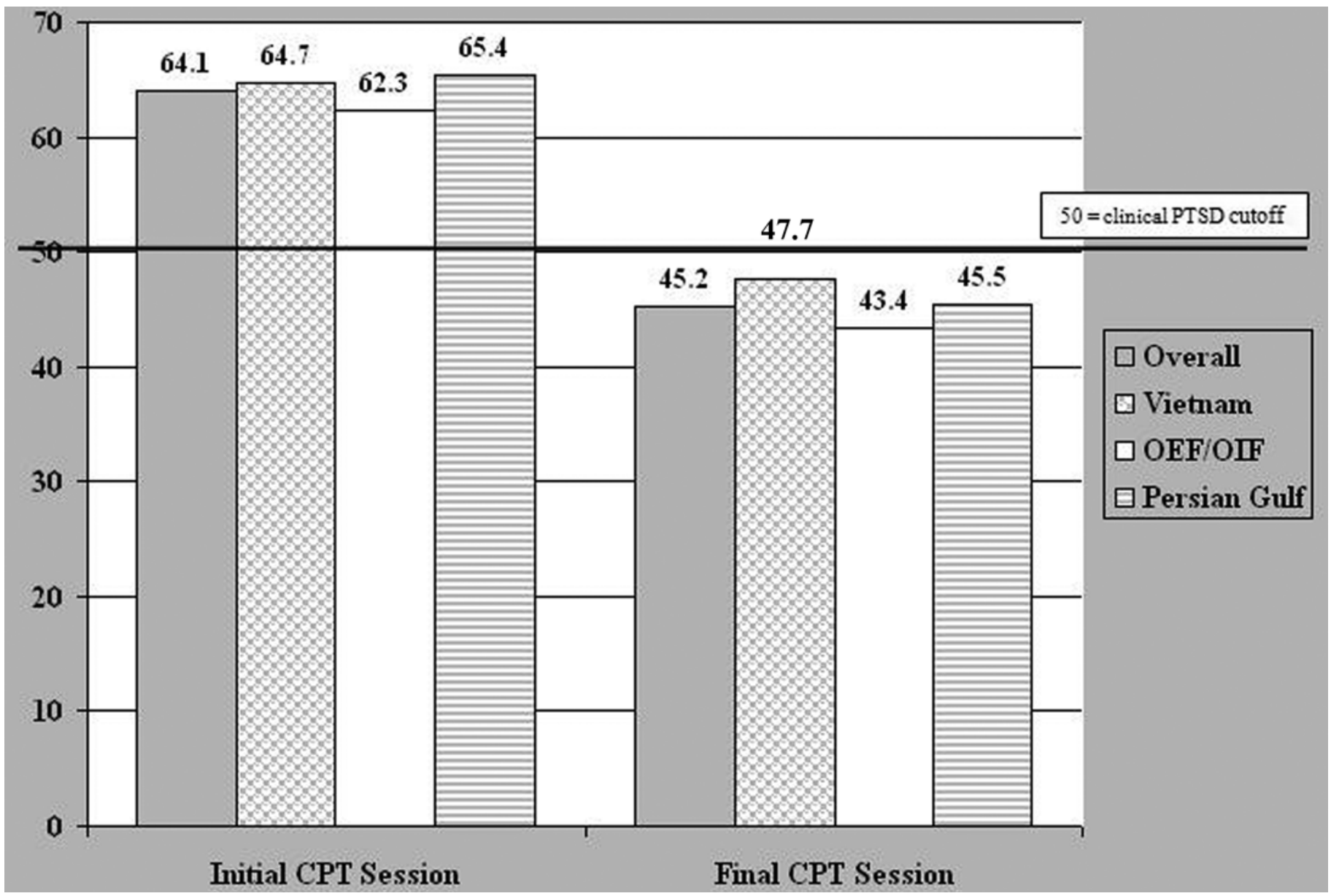

Figure 1.

Change in Posttraumatic Stress Disorder (PTSD) Checklist scores from initial therapy session to final therapy session for all cognitive processing therapy (CPT) completers. OEF = Operation Enduring Freedom, OIF = Operation Iraqi Freedom.

expertise available at regional and local levels to broaden dissemination and promote sustainability.

In addition to the administration changes, supplementary clinical education resources were developed to further enhance clinicians' understanding of the treatment and their confidence and self-efficacy in implementing CPT. A manual was created to serve as a clinical and administrative resource for consultants and to standardize the consultation processes. More specifically, the manual addresses concerns about comorbidity and patient complexity, discusses treatment rationale, and identifies ways to enhance patient motivation. To help clarify several of the key treatment concepts, such as stuck points, new help sheet guides for therapists and patients were developed to simplify complex concepts. To further enhance training efforts, additional video vignettes were devel- oped to further demonstrate the implementation of CPT with individuals and groups at training workshops. In addition, bimonthly Live Meeting tutorials (live lecture by conference call accompanied by slides) are conducted on a variety of treatment-related topics, led by VA CPT trainers. Finally, we have launched the CPT Online Enhancement Course, which is a comprehensive, interactive Web-based course for VA clinicians to refresh their CPT knowledge, review CPT concepts, and view video clips of workshops and sample therapy sessions.

\section{Using Cognitive Processing Therapy in Clinical Practice}

As the empirical and effectiveness research suggests, CPT can be used to treat PTSD and related symptoms (e.g., depression, guilt, dissociation, anger, and cognitive 


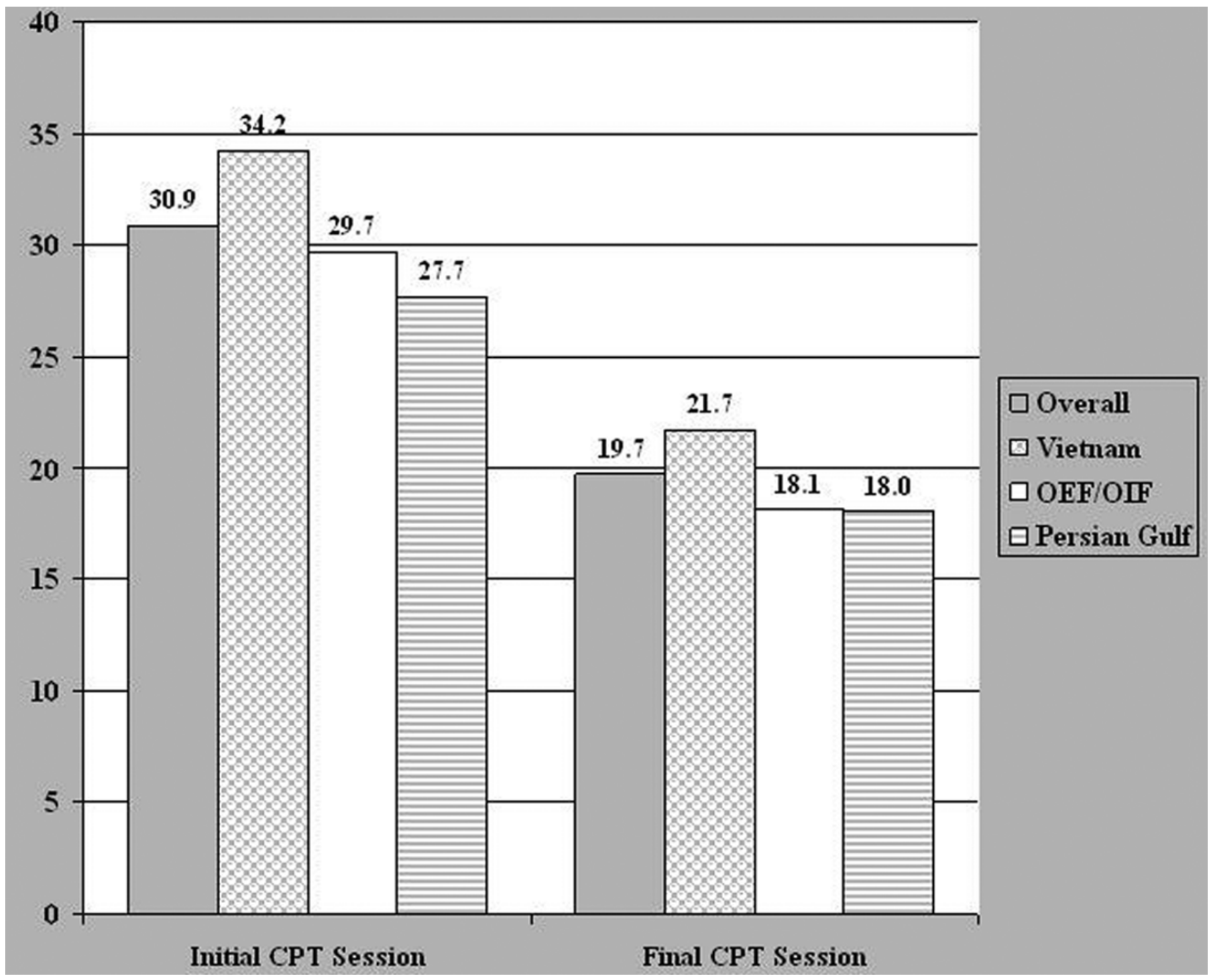

Figure 2.

Change in Beck Depression Inventory-II scores from initial therapy session to final therapy session for all cognitive processing therapy (CPT) completers. OEF = Operation Enduring Freedom, OIF = Operation Iraqi Freedom.

distortions) for individuals who have experienced a variety of traumas, including child abuse, rape, and combat. In addition, all of the CPT clinical trials discussed previously included patients with active substance abuse and this factor was not found to affect treatment outcome when analyzed in RCTs. Thus, CPT can be implemented with a wide range of patients seen frequently in clinical practice, using individual, group, or combined group and individual formats, once or twice a week. Even so, there are a few conditions in which CPT should not be initiated, including with patients who are actively psychotic, those with unmedicated bipolar mania, those with active suicidal or homicidal intent, and those in need of substance dependence detoxification. In addition, while CPT can be used with patients with cognitive limitations, individuals with severe dementia and those who are completely illiterate would not be as appropriate for CPT because of the reliance on written homework assignments. As noted previously, there has only been one study comparing CPT with another evidence-based treatment (PE); thus, it is too early to say which patients are more appropriate for one treatment over the other [2]. 
Attempts are currently being made to replicate this comparison study with Veterans, and if completed, this information will help to inform clinicians as to which therapy may work better for which type of patient.

\section{CONCLUSIONS}

CPT is a well-established treatment for PTSD, and the VA dissemination of CPT has significantly increased Veteran access to evidence-based treatments for PTSD. Initial data suggest that most clinicians who have been trained in CPT have adopted it as part of their standard clinical practice, and the patients they treat are showing solid improvements in their PTSD and depressive symptomotology. Future areas of focus for CPT dissemination in VA will include expanding decentralized training and consultation capacity and evaluating the effectiveness thereof; implementing supplemental training resources for solidifying and expanding learned skills, such as deploying the CPT online enrichment course; and promoting sustainability. In addition to continued efforts by the dissemination team, there are currently more than a dozen funded RCTs of CPT examining issues such as group versus individual CPT, CPT with OIF/OEF combat Veterans, variable length CPT, CPT delivered through telehealth, CPT with or without TBI, and CPT in combination with other treatments for smoking or alcohol abuse. We anticipate that additional data from these studies, as well as clinic-based effectiveness studies (e.g., Alvarez et al. [8] and Gilman et al. [14]), will be available over the next several years that will shed further light on the effectiveness of CPT for a variety of populations and traumas. Continued research on and evaluation of the effectiveness of CPT, the mode of delivery of CPT, and real-world implementation of CPT will improve the quality of care for PTSD and evidence-based treatment accessibility for all Veterans.

\section{ACKNOWLEDGMENTS}

\section{Author Contributions:}

Study concept and design: K. M. Chard, B. E. Karlin, P. A. Resick. Acquisition, analysis, and interpretation of data: K. M. Chard, E. G. Ricksecker.

Drafting of manuscript: E. T. Healy, E. G. Ricksecker, K. M. Chard. Critical revision of manuscript for important intellectual content:

B. E. Karlin, K. M. Chard, P. A. Resick.

Statistical analysis: E. G. Ricksecker.
Obtained funding: P. A. Resick, K. M. Chard, B. E. Karlin. Administrative, technical, or material support: K. M. Chard, E. T. Healy, E. G. Ricksecker.

Study supervision: K. M. Chard.

Financial Contributions: The authors have declared that no competing interests exist.

Funding/Support: This material was based on work supported by the VA Office of Mental Health Services.

Participant Follow-Up: Clinicians will not be individually contacted, but the CPT Web site will have the results available to clinicians participating in the rollout.

Disclaimer: Contents of this manuscript do not reflect the opinions of the U.S. Government or VA.

\section{REFERENCES}

1. Resick PA, Schnicke MK. Cognitive processing therapy for sexual assault victims. J Consult Clin Psychol. 1992; 60(5):748-56. [PMID:1401390] http://dx.doi.org/10.1037/0022-006X.60.5.748

2. Resick PA, Nishith P, Weaver TL, Astin MC, Feuer CA. A comparison of cognitive-processing therapy with prolonged exposure and a waiting condition for the treatment of chronic posttraumatic stress disorder in female rape victims. J Consult Clin Psychol. 2002;70(4):867-79. [PMID:12182270] http://dx.doi.org/10.1037/0022-006X.70.4.867

3. Monson CM, Schnurr PP, Resick PA, Friedman MJ, Young-Xu Y, Stevens SP. Cognitive processing therapy for veterans with military-related posttraumatic stress disorder. J Consult Clin Psychol. 2006;74(5):898-907.

[PMID:17032094]

http://dx.doi.org/10.1037/0022-006X.74.5.898

4. Chard KM. An evaluation of cognitive processing therapy for the treatment of posttraumatic stress disorder related to childhood sexual abuse. J Consult Clin Psychol. 2005;73(5): 965-71. [PMID:16287396] http://dx.doi.org/10.1037/0022-006X.73.5.965

5. Resick PA, Galovski TE, O’Brien Uhlmansiek M, Scher $\mathrm{CD}$, Clum GA, Young-Xu Y. A randomized clinical trial to dismantle components of cognitive processing therapy for posttraumatic stress disorder in female victims of interpersonal violence. J Consult Clin Psychol. 2008;76(2):243-58. [PMID:18377121] http://dx.doi.org/10.1037/0022-006X.76.2.243

6. Chard KM, Schumm JA, Owens GP, Cottingham SM. A comparison of OEF and OIF veterans and Vietnam veterans receiving cognitive processing therapy. J Trauma Stress. 2010;23(1):25-32. [PMID:20146255] http://dx.doi.org/10.1002/jts.20500

7. Chard KM, Schumm JA, McIlvain SM, Bailey GW, Parkinson RB. Exploring the efficacy of a residential treatment 
program incorporating cognitive processing therapy-cognitive for veterans with PTSD and traumatic brain injury. J Trauma Stress. 2011;24(3):347-51. [PMID:21626573] http://dx.doi.org/10.1002/jts.20644

8. Alvarez J, McLean C, Harris AH, Rosen CS, Ruzek JI, Kimerling $\mathrm{R}$. The comparative effectiveness of cognitive processing therapy for male veterans treated in a VHA posttraumatic stress disorder residential rehabilitation program. J Consult Clin Psychol. 2011;79(5):590-99.

[PMID:21744946]

http://dx.doi.org/10.1037/a0024466

9. Karlin BE, Ruzek JI, Chard KM, Eftekhari A, Monson CM, Hembree EA, Resick PA, Foa EB. Dissemination of evidence-based psychological treatments for posttraumatic stress disorder in the Veterans Health Administration. J Trauma Stress. 2010;23(6):663-73. [PMID:21171126] http://dx.doi.org/10.1002/jts.20588

10. Weathers F, Litz B, Herman D, Huska J, Keane T. The PTSD Checklist (PCL): Reliability, validity, and diagnostic utility. Annual Meeting of the International Society for Trauma Stress Studies; 1993 Oct; San Antonio, TX.

11. Beck AT, Steer RA, Brown GK. Beck Depression Inventory-II manual. 2nd ed. San Antonio (TX): Psychological Corporation; 1996.
12. American Psychiatric Association. Diagnostic and Statistical Manual of Mental Disorders. 4th ed. Washington (DC): American Psychiatric Association; 2004.

13. Beck AT, Steer RA, Ball R, Ranieri W. Comparison of Beck Depression Inventories -IA and -II in psychiatric outpatients. J Pers Assess. 1996;67(3):588-97.

[PMID:8991972] http://dx.doi.org/10.1207/s15327752jpa6703 13

14. Gilman RC, Schumm JA, Chard KM. Hope as a change mechanism in the treatment of posttraumatic stress disorder. Psychol Trauma. 2012;4(3):270-77. http://dx.doi.org/10.1037/a0024252

Submitted for publication October 21, 2011. Accepted in revised form March 1, 2012.

This article and any supplementary material should be cited as follows:

Chard KM, Ricksecker EG, Healy ET, Karlin BE, Resick PA. Dissemination and experience with cognitive processing therapy. J Rehabil Res Dev. 2012;49(5):667-78. http://dx.doi.org/10.1682/JRRD.2011.10.0198

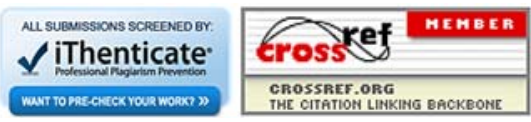

